Historic, archived document

Do not assume content reflects current scientific knowledge, policies, or practices. 



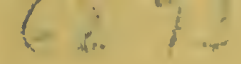

46 

$1845-46$.

D. ANDR돈 \& FUITON口

A I I D G E D

- anNual catalogue

or

HOT-HOUSE AND GREEN-HOUSE PLANTS,

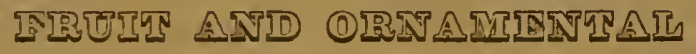

TREES, SHRUBS, \&c. \&c.

CULTIVATED AND SOLD

AT THE OLDESTABLISHED NURSERIES,

\section{LATE OF}

D. \& C. LANDRETH,

FEDERAL STREET NEAR TIE ARSENAL,

(CITY OFFICE, 65 CHESNUT STREET, )

PII IAA D ELPHIA. -

\section{poniladolphía:}

PRINTED BY STAVELY \& MCCALLA,

No. 12 Pear street.

\section{5.}




\section{ADVERTISEMENT.}

The popularity of this establishment for over half a century, and the support which has been extended it by every section of the Union, but especially the Middle, Southern and Western States, would seem to render appropriate some expression of our thanks on the issue of another edition of our Catalogue ; which at the same time, affords opportunity to make known our determination to do as we always have-consider the purchaser's interest and our own as nearly connected.

The collection existing at the Nursery is now so diversified that a catalogue in detail would be too voluminous for general distribution, and we have been compelled therefore to confine the present edition to the more prominent articles, and those of general interest. Purchasers of rare plants will find at our establishment a large collection, not included herein, which they are invited to examine.

The fruit department contains a careful selection, purposely limited in variety, of the more desirable fruits; and additions are annually made of such new ones as prove worthy. The utmost vigilance is exercised in preserving the respective tarieties distinct-all the trees being "worked" under their personal inspec- $/ 1 / 1$ " tion; purchasers may therefore rely on obtaining the identical valiety represented.

The prices for Ornamental Trees and Plants have been greatly reduced. Where the prices vary, it is in consideration of size, form, \&c.; in such cases the order had better indicate the cost. To obviate misunderstanding, it may be proper to premise that no care will be spared in the removal and package of all orders intrusted to us, but no responsibility will be incurred as to their safe carriage or ultimate success.

The terms are CASH, or REsponșible City reference.

A 1 liberal discount allowed to wholesale purchasers.

D. LANDRETH \& FULTON.

Philadelphia, July, 1845. 


\section{HOT-HOUSE PLANTS.}

Botanic Names.

Alstromoria ligtu

Aristolochia labiosa

Astrapar Wallichii

Begonia inaculata

Billbcrgia pyrainidali

Bletia tankerviltix

Burchelia capensis

Bonapartia juncea

Buddlca madagascaricnsis

Calatlıca Zcbrina

Coffca arabica

Combretum purpurea

Crinum Amabilc

Crassula coccinnea

Cypripedium insigne

Draccna ferrea

Euphorhia splendens

$$
\begin{aligned}
& \text { jacquinicellora } \\
& \text { Poinsettii }
\end{aligned}
$$

Frantlicmum pulchella

Ficus macrophylla clastica

Gesncria magnifica spicata

Gloxinia mactilate candida hirsuta speciosa. sanguinca spcciosa-alba caulescens

IIclitropiun, intermedia

Hibiscus rosa-sinensis variegato plenus lutea plenus carneo plenus rubro plenus liliiflorus

Hoya carnosa

Ixora coccinnea

Jasminum sambac

Justicia coccinnca

$$
\begin{aligned}
& v \text { a pleno } \\
& v \text { trifolialum }
\end{aligned}
$$

Iaurus cinnamomum

Melastoma hetcromalla
Cominon Names. $\$ \mathrm{cts}$. क cts.

stripe flowercd..... 50

spotted... . ..... $100 \quad 300$

splendid .........2 00500

spotted .......... 25

rosc coloured ..... 75

Lady 'Tankervills . . 100200

capc ........... 75

ruslı lcaved ... ....2 2001000

orange flowered ... $50 \quad 100$

zebra plant....... I 00300

collec tree........ $100 \quad 200$

scarlet flowering.... 100

splendid ......... 100500

scarlct.......... 50

ladics' slipper...... 100200

purplc leaved ...... ; 00

scarlet profuse..... $50 \quad 250$

orangc-verybeautiful $50 \quad 200$

large flowering..... $50 \quad 300$

blue........... 30

large leaved... _.. $100 \quad 500$

Indian rubber..... 50500

crimson......... 100300

profuse flowering... $100 \quad 300$

spotted.......... 50

pure white ...... 50

hairy ......... 50

bluc.......... 50

scarlet ........ 50

pale white....... 50

steinmy......... 50

finest ........ . $25 \quad 50$

single red. ....... 50

double striped...... 50

do buff ....... 50

do salmon ..... 50

do red........ 50

lily flowered....... 50

wax plant......... 50

scarlet flowering.... $50 \quad 100$

Arabian jasmine.... $50 \quad 100$

semi-doublc ....... 50

Tuscan, very double $100 \quad 300$

scarlet.......... 50

cinnamon........ 100500

large leaved ...... 50 
Rotanic Names.

Myrtus tomentosa

Musa, several varieties

Passaflora Loudonia

raeemosa

alata

Russelia juneea

Rhipsalis pendula

Tabernamontana coronaria
Common Names.

$\$ \mathrm{cts} . \$ \mathrm{cts}$.

Chinese myrtle... . 50100

crimson ... ..... $50 \quad 500$

red .......... 50100

winged......... $50 \quad 100$

crimson ........ 50

weeping........ $50 \quad 100$

fragrant ........ $50 \quad 100$

\section{GREEN-HOUSE PLANTS.}

Acacia cinerescens verticillata

armata

Agapanthus umbellatus

Aloe lingua

variegata

Aloysia citri-odora

Alstromeria pelcgrina pulehela.

Amaryllis undulata Jolinsonii purpurca. aulica

Arbutus andraehne unedo

Ardesia crenulata Aster argophyllus Aucuba japonica

Azalea indica elcgans

latcritia variegata Danielsiana purpurea pleno alba phonccia Gillinghami nova blanca copeii sinensis

Banksia serrata Bignonia pandora Bouvardia triphylla Burchellia capensis Buxus balearicus

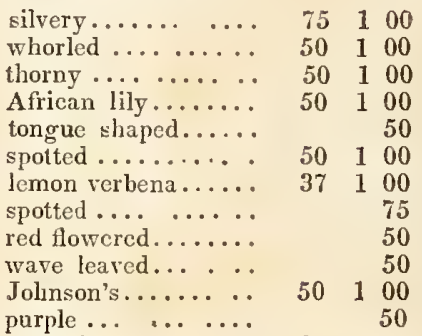

splendid ... ...... 100

snw lcaved ....... 100

common... ..... 100

crenulated ....... $50 \quad 100$

musk seented ..... $50 \quad 100$

Japan gold tree.... $50 \quad 100$

red flowered..... $25 \quad 300$

lilac... ... ... $25 \quad 300$

salmon eolour..... $50 \quad 200$

rose red and white.. 50200

red........... 50200

double purple..... $25 \quad 500$

white.......... $25 \quad 500$

purple . $\quad \ldots \ldots . .25 \quad 2500$

lilac.......... $37 \quad 500$

purc white...... 25200

rose .......... $37 \quad 300$

Chinese yellow .... 100

saw leaved....... 200

eluster flowering.... 100

scarlet........ 37

cape........... $50 \quad 100$

Spanish box....... $50 \quad 100$

\section{CACTII.}


Botanic Names,

Cercus Cyliniricus

Mallisonia

Sinithii

flagelliformis

horridia

gladiuta

geinmaitus

spcciosissimus

heptagonus

Epiphyllum speciosa

splendens

Jenkinsonta

truncatus

Russcllianus

atropurpurca superba

vandesii

Manmillaria prolifera

ivildcana

rhodantlia

stellata

radiata

rubra

Eclinocactus Eyrcisii

decora

ottonis

dis-color

simplex

pulchella

Opuntia, microdysa

leuacantlua
Cummon Names. \$ cts. \$ cts.

round ........ $50 \quad 500$

scarlet.......... $50 \quad 200$

scarlet......... $50 \quad 200$

crceping....... $\quad 25 \quad 100$

spiney.......... $50 \quad 100$

strong spined ...... $50 \quad 100$

crect........... . $50 \quad 100$

splcudid ........ $25 \quad 300$

.............. 25100

purple......... 25200

crimson......... 25200

dark crimson..... 25200

crimson ....... 25200

purplc $\ldots \ldots \ldots \ldots .25200$

25200

25200

$25 \quad 200$

25200

50200

25100

25100

25100

25300

50300

25300

50300

50300

$\begin{array}{lll}50 & 2 & 0\end{array}$

50100

50100

\section{CAMELLIAS.}

Particular attention is given to the cultivation of this splendid genus; our collcction enbraces some two thousand grafted plants of every desirable varicty, the lowest price designated is for well established plants, 6 to 12 months cnarched. Also, 2000 Camcllia Stocks, varying in price from $\$ 10$ to $\$ 25$ per lundrcd, according to size.

Those marked * are of American origin.

Camcllia alba.pleno

simplcx

fimbriata

* Americana.

aninconiflora

rosca

alba

althxiflora

atrorubens

* amibile

- Binneyii double white..... 1502000 single, do........ $100 \quad 500$ fringed, do... .. 1501500 bluslı rose spot.... 150500 red warrataw..... $100 \quad 1000$ rose............ 1501000 whitc ......... 1501000 crimson........ 100500 red ............ $100 \quad 500$ rose and red...... 1001000 liglıt rose........ 300 
Botanie Names.

Camellia Bealii

Carswelliana

Chandleri

* Caroline

Colvelleii

coneinna

conchafiora

celestina

candidissima

conspicua

carnea

Camplellii

Donklaarii

Elphinstonia

cxemia

*Estherii

elegans

clata

eclipse

* Floyii

Flordii

Fairlea

fulgida

frank furtensis

Florida

* Feastii

Gilosii

Gunnelli.

gaussonia

${ }_{*}^{*}$ Grahamii

*Hosackii

Hendersonia

* Hempsteadii

Henry Favre

imbricata

alba

incarnata

Invincible (Grey's)

Juliana

japonica

Kingii

Kermosina

*Landrethii

mutabilis

myrtifolia

mutabilis triversi

nivalis

ochraleuca

oleafera

Parksii

* Philadelphia

*Prattii

pomponia
Common Names, \$ ets. $\$$ ets. large red....... $150 \quad 1000$ dark red.......... \& $50 \quad 1000$ white and crimson. . $150 \quad 1000$

whitc rose stripe.... $150 \quad 1000$ dark red ......... $150 \quad 1000$ light $\operatorname{rose} \ldots \ldots \ldots$... 00500 rose............ $100 \quad 500$ purest white....... 1501000 large red......... 1001000 pink......... 1001000 white and red..... $150 \quad 500$ white rose \& crimson $150 \quad 1000$ red and wlite.... $100 \quad 500$ salmon.......... $100 \quad 500$ white and rose... $150 \quad 500$ rosc........... 1501000 crimson......... 1501000 white rose stripe.... 150 I0 00 rose red....... 1501000 dark rose........ 150 I0 00 crimson ......... 1501000 crimson .... ... .. 10000 $\operatorname{rosc} \ldots \ldots \ldots \ldots \ldots . .1001000$ red ............ $100 \quad 1000$ white and rose... 400 crimson and whitc.. $150 \quad 500$ white........ 300 pink........... $100 \quad 500$ white......... $150 \quad 500$ crimson ......... 1001000 rose............ 1001000 dark rose....... 300 rose ..... .... $150 \quad 1000$ crimson and white.. 1501000 white and rose..... 1501000 Lady Humes' ...... 1501000 rose, red spots...... $150 \quad 500$ whitc.......... $100 \quad 500$ single red... .... $50 \quad 500$ white rose spot.... $150 \quad 500$ crimson......... 1001000 rose and whitc. .. 1502000 cliangeable crimsonı. 1005000 light red ......... $150 \quad 100$ rose............ 1501000 white ... ...... 1001000 white .......... $100 \quad 500$ white......... 1501000 red and white .... $150 \quad 500$ rose red. ........ 1501000 light $\operatorname{rosc} \ldots \ldots \ldots 1501000$ white ... ...... $100 \quad 500$ 
Botanic Names,

Cauncllia pendula proniaflora

Qucen, (Fielder's)

reticulata

rosa-sinensis

rosea

Rex Batavia

rosa-mundi

rubro-pleno

sasanqua rusea

sabina

Swectii

speciosa

spicata

Cunninglam's

*Shcrwoodi

tricolor

triumphans

thea

vandesia superba

varicgata

viridus

welbankiana

William the 4 th

* Waslington

Wardii IVoodsii

Cliamxrops hystrix

Cherianthus plene

Cincraria crucutar kingi

Clianthus puniccns

('itrus modica

nobilis

limonia

bergamense

aurantia

Cobea scandens

Correa speciosa

Crassula coccinna

Cratwgus glabra

Crinum amcricana

Cycas revoluta

Cyclamen persicum

Dapline odora striatum

Dianthus caryoplyyllus

(several handsome varieties)
Common Names. \& cts. \& cts.

rose ........... $100 \quad 500$

rose ......... $100 \quad 500$

light rose $\ldots . . .200$

rose........... I $50 \quad 500$

rose............ 1001000

rose purple....... $150 \quad 500$

white rose striped.. $150 \quad 500$

rose and white.... 1001000

old red......... 1101000

light rose...... $150 \quad 500$

rosc........... $100 \quad 500$

rose spotted with red $150 \quad 1000$

crimson and whitc.. $100 \quad 1500$

rose and white..... $100 \quad 500$

red............ 1001000

crimson and white.. $150 \quad 500$

whitc rose \& crimson $150 \quad 500$

rose spotted white. 1501000

black tea........ $50 \quad 100$

crimson ........ $100 \quad 500$

rose and white... $150 \quad 1000$

green tea....... $50 \quad 100$

grecnish white .... $100 \quad 1000$

rose spotted with whitc 2001000

400

crimson ......... 1001000

$\operatorname{rosc} \ldots \ldots \ldots \ldots \ldots 1001500$

lıcdgc-hog palm ....2 $200 \quad 500$

double wallflower.. 2500

purple leaved....... 3700

splendid......... 3700

scarlet.......... $50 \quad 100$

citron trce....... ] $00 \quad 500$

Chines, dwarf or-7

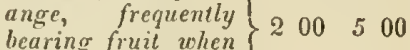

10 to 12 inches high J

lemon ........ 1003000

Bergamottemon . . 1001500

scville orango .... $100 \quad 300$

elimbing.......... 3700

red flowering .... 200

scarlet......... 50

white......... $50 \quad 100$

Amcriean .... ... $50 \quad 300$

sago palm........ $100 \quad 500$

whitc ........ $50 \quad 500$

fragrant....... $\quad 50 \quad 100$

striped ........ $50 \quad 100$

$\{$ earnation pink.... 
Botanic Names.

Lpacris grandiflora

Erytherina crista galli

Eucomus punctata

Eugenia jambos

Escallonia rubra

Fuchsia coccinnea

$$
\text { globosa }
$$

longiflora

Gardenia florida radicans

Gladiolus formosus and ? other varictics.

Gorteria pavonia

Hydcrangia hortensis

Illicium floridanum

Jasminum grandiflora

Kennedia prostratra

Lagerstroemia indica

Laurus camphora

Leucadendron argentea

Linum tryginum

Lechenaultia formosa

Loniccra japonica

Magnolia fuscata

Myrtuz communus

Manettia cordifolia

Ncrium:splendens

Olea Europca alba simples fiagrans

Passaflora princeps coerulca
Common Names.

large flowering....... cocks-comb tree...

spotted... ... ...

rose applc..........

red ... ........

eardrop...........

globular ........

large..........

cape jasmine ......

dwarf ... ... ...

sword lily.........

spotted ..........

changcable .......

aniseed..........

catalonia........

prostratc........

crapc flower . ....

camphor trcc......

silver tree $\cdots \cdots \cdot . \cdot$.

yellow .........

scarlet ........

japan.........

rusty leaved .......

myrtlc..........

scarlet...... ...

variegated oleander..

single white.......

olive............

fragrant ........

scarlet... ......

bluc...........
$\$$ cts. cts.

50100

50100

50

$\begin{array}{llll}1 & 00 & 3 & 00\end{array}$

50

37

37

50

$\begin{array}{lll}37 & 1 & 00\end{array}$

$\begin{array}{lll}37 \quad 1 & 00\end{array}$

50200

25100

$\begin{array}{lll}50 & 1 & 00\end{array}$

$\begin{array}{lll}50 & 1 & 00\end{array}$

50

$\begin{array}{lll}50 & 5 & 00\end{array}$

$\begin{array}{lll}50 & 1 & 00\end{array}$

$200 \quad 300$

50

50

50

$50 \quad 100$

$25 \quad 100$

50

$\begin{array}{lll}50 & 1 & 00\end{array}$

$\begin{array}{lll}50 & 1 & 00\end{array}$

$\begin{array}{lll}50 & 1 & 00\end{array}$

$50 \quad 100$

50

50

pelargoniums-50 Cents each. aglaia

Bridcgroom

Cassius

Coronation

Champion

Duchess of Roxbury

Beatrice

Corinne

Matilda

Ophelia

Orange boven

Garth Fanny

Gem

Jewess

King

Lifeguardsman

pink

large blush

crimson

salmon

large rose

fine rose

rosy blush

rose

rosy crimson

light rose

orange red

pink

rosy red

rosy red

crimson

rose 
Botanic Nimes.

Pclargonium Madonna

Perfection (Gartl's)

Quecn of Scots

Victoria

Sylph, and many other fine varietics
Pittosporum tobira

Plumbago capensis

Polianthus tuberosa

Rlıodochiton volubilus

Rlododendron arborea

Salvia splendens ponticum

Stapeliit anbigua

Strclitzia regiua

Tecoma capensis

'Tigridia paronia

Verbena-15 sclect varictics
Common Names, \& cts. \& cts. white

dark rose

crimson

rosy pink

$\{$ pink. frogrant. ......... $25 \quad 200$

bluc........... $25 \quad 100$

double, per dozen.... 100

purple-splendid... $\quad 25$

trce mountain laurel 2001000

Pontic.......... $100 \quad 500$

searlet........... 25

star flower....... $50 \quad 100$

bcautiful ........2 200 1000

cape.......... $25 \quad 2500$

tiger flower, per dozen $\quad 100$

\section{ORNAMENTAL TREES.}

Acer dasycarpum nergundo psuedo platanus saccharinum platanoides

Esculus pavia ohioensis lippocastanum macrostacliya

Ailantlus glanciulosa

Alnus glutinosa

Aralia spinosa

Anona triluba

Betula nigra

Broussonetia papyifera

Castanca visca

Catalpa cordifolia

Celtis occidentalis

Cercis canadensis

Chionanthus virginica

Cornus mascula Horida

Cupressus disticha

Cytissus laburnum pendula silver leared maple.. 50100

ash leaved......do.. 50

curopean sycamore.. $50 \quad 200$

sugar maple....... $50 \quad 100$

norway, do...... 50

scarlet horse cliestnut 100

buck-cye......... $50 \quad 100$

curopean........ $50 \quad 200$

dwarf whitc....... $37 \quad 100$

trec of Hcuven..... $50 \quad 100$

cnglish alder....... $50 \quad 100$

prickly ash........ 50

pawpaw......... 50

black birch........ $50 \quad 100$

paper mulberry.... . 30100

spanish chestuut.... 50

candle tree........ 50

ncttle trec........ 50

judas trec........ 50

fringe tree.... ... $50 \quad 100$

yellow dogwood.... 50

white do...... 50

deciduous cypress.... $50 \quad 100$

golden chain....... $25 \quad 50$

wecping........ 100 
Botanic Names,

Fagus sylvatica

americana

Fagus pendula

purpurea

asplenifolius

Fraxinus americana striata excelsoir

Gordonia pubescens

This is a very ornamental tree, covered with white flowers from midsummer until frost.

Gymnocladus canadensis

Hyppophea rhamnoides

Halcsia tetraptera

Kolrcuteria paniculata

Liriodendron tulipifera

Larix europea

americana

Magnolio aucuminata

glanca

tripetala

macropliylla

conspicua

purpurea

Maclura aurantiaca

Pynckueya pubens

Platanus occidentalis orientalis
Common Names. \$cts. \$ cts.

european beech..... $50 \quad 100$

american, do...... 100

weepirig, do...... 100

purple-(very dark).. $100 \quad 200$

fern leaved........ 100

whitc ash........ $50 \quad 100$

silver striped do..... 100

weeping do..... $100 \quad 200$

Franklinia ...... $50 \quad 200$

Kcntucky coffee.... $\quad 50 \quad 100$

silvery......... $25 \quad 50$

snowdrop or silver bell $\quad \mathbf{5 0}$

clinese......... $50 \quad 100$

tulip poplar....... 50 I 00

european......... $50 \quad 100$

hakmatuk ....... $50 \quad 100$

cucumber trce..... $50 \quad 150$

swect scented..... $50 \quad 100$

umbrella tree..... $50 \quad 100$

large flowering..... $100 \quad 300$

chandelier-beautiful $100 \quad 500$

purple - shrubby... $50 \quad 200$

osagc orange...... $50 \quad 100$

Gcorgia bark tree.. $\quad 100$

american buttonwood $\quad 50$

Asiatic.....do....... $50 \quad 100$

PAULONIA MPERIALIS-price 1 each.

This Trec is of recent introduction, is very ornamental and excecdingly rapid in growth, we liave seen the leaves twenty inclues in diameter, it is a wative of the East Indies and pcrfectly bardy:

Populus alba

niveum

candicans

tremula

Prunus serotina padus

Qucrcus alba

phellos

palustris

macrocarpa

Rlıammus catharticus

Rhus cotinus

Salisburia adiantifolia

Salix babylonic annularis

Sorbus aucuparia americana ábele-poplar.... . $\quad 50$

new white leaved.... $\quad 50$

atheniun........ 50

aspen.......... 50

awerican bird cherry 50

english bird cherry.. $\quad 50$

white oak........ $50 \quad 100$

willow........... $50 \quad 100$

swamp... ....... $50 \quad 100$

cork bark........ 50

buck thorn......... 37

venetian sumach..... $25 \quad 50$

Ginko tree...... 100

weeping willow... $50 \quad 100$

ring leaved....... $50 \quad 100$

roan tree ......... 50

mountain ash..... $50 \quad 75$ 
Bolanic Names.

Shepherdia argentea

Tilia amcricana rubra europea

Uluus americana inontana canpestris
Common Names.

Buflalo berry ...... american bass-tvood. red twig.......... yellow twig......... american $\mathrm{clm} . . .$. wych .... do ..... english .........
$\$ \mathrm{cts} . \$ \mathrm{cts}$ 50

100

50100

50100

$\begin{array}{lll}50 & 1 & 00\end{array}$ 50

\section{ORNAMENTAL SHRUBS.}

Acacia julibrissin

Azalea ealendulacea nudatlora

Pontica

Sincusis

Amorplua fruticaus

Amygdalus pumila

Aucuba japonic'a

Calycanthus floridus l'cunsylvanicus glatcus

Chimonanthus fragrans

Clethri alnifolia

Colutea arborescens

Corchorus japonica fluruplenus

Cratægus oxyccantha rubro

Cydonia japonica alba

Deutzia scabra

Dirca palustris

Euonymus americanus curopous

libiscus alba plenus purpurcus do varicgata varicgata

Hyderangea quercifolia hortensis

Jea rirginica.

Jasminum ofticinalis

Philadelplus nanus

grandiflorus

inodorus

Gordonianıs

Ptelca trifoliata

Robinia hispida

Ribeg aureum silk tree......... 50100 american wood loncy suckle 50 pink ........... 50

light orange....... 50

chincse yellow..... 100

indigo shrub. ..... 50

alinond, double flowering $\quad 25$

gold trec........... 50

swcet secnted shrub. $\quad 25 \quad 50$

large flowering... .. $25 \quad 50$

dark do....... $25 \quad 50$

chinese alspice..... 75

alder lcaved....... 25

bladder senna....... $\quad 37$

single yellow...... 25

double do ... ... 25

European thorn...... 25

red ilowering....... 50

red pyrus........ $50 \quad 100$

white do ..... 75100

French moek orange. . $\quad 37$

leatlicrwood ...... 25

burning busl....... 37

curopean...... . . 37

double white Altlica. $\quad 50$

do purple....... 37

painted lady...... $\quad \mathbf{3 7}$

striped leaved....... 50

oak leaved........ $25 \quad 50$

cliangeable......... $25 \quad 75$

whitc flowered..... 25

white do....... 25

dwarf mock orange.. $\quad 25$

white flowcring..... 25

sccntless ....... 25

Gordon's ......... . 50

trefisil.......... 37

rose acacin........ $\quad 50$

Lewis' fragrant...... $\quad 25$ 
Botanic Names.

Spirea montana betulfolia hypcricifolia tomentos $x$ sorbufolia

Stuartia Marylandiea

Styrax lævigita

Symphora racemosa

Syringa alba vulgaris

Persica purpurea laciniata purpurea

Tamarix gallica

Vibirnum opulus rosea
Common Names. \$ cts, \$ cts. mountain spirea..... birch lcaved... ... . hypericum leaved.... downy ............. sorbus leaved....... large flowering...... smooth leaved........ snow-berry ........ white lilac........ Persian... ....... parslcy leaved.... . common purple ..... tamarisk.... ....... snowball... . ... 25
25

\section{EVERGREEN TREES.}

Cedrus lebani deodora

Cupressus thuyoides

Juniperus virginica succica

Prunus lauro-cerasus

Pinus pinaster

pinea

strobus

balsamea

picea

canadensis

excelsa

sylvestris

alba

Frazieri

Douglassi

Webbiana

Morinda

pichta

Taxus baccata hibernica

Thuja occidentalis orientalis
Cedar of Lebanon..

100

100

whitc cedar....... 50

red cedar........ 25

swedish juniper....

english laurel......

cluster pine ......

Italian stone ......

whitc or silver......

Balm of Gilead...... european silver fir..

Hemlock sprucc.....

Norway.... . .....

scotch...........

Norway white, beautiful

Fraziers.........

37100

37

100

100

$\begin{array}{lll}50 & 1 & 00\end{array}$

$50 \quad 500$

$\begin{array}{lll}50 & 2 & 00\end{array}$

50200

50200

$\begin{array}{lll}50 & 1 & 00\end{array}$

75100

100

100

500

200

200

english yew...... 50 1 00

irish........... 100

amcrican arborvitae 50200

elinese......... $25 \quad 100$

\section{EVERGREEN SHRUBS.}

Buxus sempervirens

arborescens

arg variegata common box....... $25 \quad 50$

foredging walks, per yard $12 \frac{1}{2}$

green trce box...... $25 \quad 500$

silver striped .... $25 \quad 500$ 
Botanic Names.

Ilcx opaca acquifolia

* ferox

* arg varicgata

* aur do.
Common Names.

common hollcy.....

curopean .........

hedgc.hog.......

silver edged........

gold edged....... s cts. \& cts.

$25 \quad 75$

50

100

100

100

Those marked," require a slight protection during winter.

Kialmia latifolia

Magnolia grandiflora

Mespilus pyracantlia

Rliododendron maximum

pouticum

punctatum

Taxus procumbens laurcl leaved ......

cvergrecn magnolia. . scarlet berriced.... mountain laurel.... purplc........... spotted. . ... ... ancrican yew...... 50

$\begin{array}{rrr} & & 50 \\ 50 & 1 & 00 \\ 55 & & 75 \\ 1 & 2 & 00 \\ 1 & 2 & 00 \\ 1 & 2 & 00 \\ 37 & 1 & 00\end{array}$

\section{VINES AND CREEPERS.}

Suitable for walls, pillars, arbors, \&c.

Ampclopsis hederacca

Aristolochia siplio

Bignonia radicans grandaflora

crucigera

Clcmatis virginica flammula

florida fl. pleno vitccolla pulchclla

Decumaria scandens

Gclscmium nitidium

Glycine frutesecns sinensis

Hedera lıclix

hibernica poctica

Jasminum cfficinale

Loniccra belgica

flexuosa

scmpervirens

Vinca major frazicrii

minor marginata

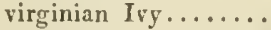

birth wort, very eurious

trumpet flower..... orange flowered, fine

cross bearing........

virgin's bowcr.......

swect scented ......

double white........

" purple.......

climbing..........

carolina jasminc.. ..

exquisilely scented...

purple flowering.....

chincsc.............

conmon iry......

Irish do.......

pocts do.......

white jasmine . ...

monthly loncy sucklo

evergreen do..

trumpet or coral do..

ycllow do..

Percwinlklc.... . ..

comnion.........

gold cdged......
100

25 


\section{PLANTS FOR HEDGES.}

Arborvitæ, chinese evergreen....1 foot high $\$ 10$ per hundred do American do......1 " do. $\$ 10$ do. do

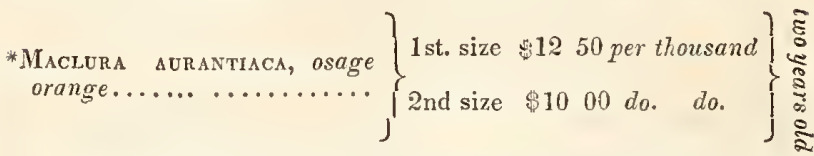

* This plant is now attracting much attcntion throughout the country, and will certainly supcrsede all others for hedging, it grows rapidly, and if regularly clipped at midwinter and midsummer rcspectivcly, will, in a few years, make a compact impenetrable fence; the rich glossy green of the foliage (which it relains longer than any other deciduous tree) makes it very ornamental, and the numerous strong, rigid thorns with which it is armed, is a certain preventive against depredation.

It delights in a moderately rich soil, and one foot apart is the pro. per distance for planting. The maclura is undoutcdly the cheapest hedge plant that has been introduccd into cultivation.

\section{PEONIAS.}

Botanic Names.

Pxonia arborea fl. pleno

papavaracea

officinalis

var rosea

var blush

edulus whitleyi

fragrans

humilis

humeii

tenuifolia

coralina

Common Names.

tree pæony.......

single white ......

double crimson....

rose. . . . ....

blush.. .........

double white.....

do. sweet scented

do. pink.......

dwarf rose.......

finc leaved........

cherry red.......
$\$$ cts. \& cts.

$\begin{array}{lll}50 & 300\end{array}$

$\begin{array}{lll}50 & 3 & 00\end{array}$

25

25

25

37

37

37

25

25

100

\section{EVERBLOOMING CHINESE ROSES.}

The Roses under the several heads have been selected with the greatest care from among some hundred varicties, and cmbrace the choicest known in Europe.

\section{BENGAL.}

This class of Roses is particularly hardy, enduring the severest winters of the middlc States : constant blooming and many of them very fragrant. 
50 cents each-per dozen, $\$ 3$ to $\$ 5$.

Animated

Arsnic

Augustine IIersant

Assureus

Admiral Duperre

Belle Isidore de monza violet

Bisson

Burctte

Chamcleon

Cramosie supcrieur

$\mathrm{Ccls}$ Aggrippina

Don Carlos

Eugene 13eauharnois

Fabvicr

Fenclon de Luxembourg

Grandval

Grandida

IJortensia

IIamilton

Indica alba

Jacksonia

Louis Phillipe

Iaidy Warrander

Lawrencia

La Renomme

IIarjolin

Irs. Bosanquet

Reine de I.ombardy

Triomphe de Gaud

Triomphant

Samson

Vanilla rosy blush

light rose

superb rose

dark red

dark rosc

crimson

dark rose

violet purple

rosy blush

dark red

rose changcable

rich crimson

ficsh color

dark rose

crimson

scarlet

rose

crimson

rose

light rose

dark pink

pure white

bright red

crimson

white

pink

light rose

crimson

large blush

cherry red

changeable rose

large crimson

light rose

dark rose

\section{DOUBLE TEA-SCENTED ROSES.}

50 cents each-per dozen, $\$ 3$ to $\$ 5$.

These are also everblooming. They are all exquisitely fragrant and gencrally hardy, in this latitude, in light loamy soil.

Archiduchesse Tlicresa

Aurora

Alba

Arkinto

Belle Marguerittc

Bougere

Boutrand

Bon silene

Bourbon white

blush

pure white

ficsh colour

rose

light rose

rosy blush

superb red

white 


\begin{tabular}{ll} 
Barbot & light rose \\
Camellia & white \\
Caroline & bright rose \\
Countess of Albemarle & straw colour \\
Due de Orleans & bright rose \\
Devoniensis & straw eolour \\
Devaux & blush \\
Flon & buff \\
Flaveseens & ycllow \\
Goleondi & crcamy white \\
Gigantesque de Lima & light yellow \\
Hymenea & white \\
Jaune panaehe & straw eolour \\
Lilieina & lilac \\
Lyonaise & rose \\
Lapaetole & yellow \\
Maneais & rosy buff \\
Madam Desprez & pure white \\
Niphetos & white \\
Odorata & eommon blush \\
Odoratissima & rieh blush \\
Princess Maria & blush \\
Prineess de Esterlizy & light rose \\
Strombio & blush white \\
Triomphe de Luxemborg & buff or rosy blush \\
Vietoria modeste & blush \\
\hline
\end{tabular}

\section{DOUBLE NOISETTE OR OLUSTER ROSES.}

Everblooming : flowering in immensc elusters, and growing with astonishing rapidity. They are admirably adapted for the eovering of walls, pillars, arbors, \&c. They arc all hardy in the latitude of Philadelphia, in light soils.

\section{* DENOTES DWARFS.}

50 cents each-per dozen, $\$ 4$ to $\$ 6$.
* Alba
* Amic Vibert
Bengal Lee
ereamy white
pure white
monstrosa
Cadot
Countess de Grillon
Chromatella
* Charles 10th
Conque de venus
Cour jaunc
Champneyana
Fellemberg
Gabriel
Jaune de Prez
* Julienne le sourd
ereamy blush

blush lilae
blush
yellow-superb, $\$ 2$
purple
white rose eentre
white yellow eentre
rosy white-very profuse
superb erimson
blush, fine
rosy yellow-very fragrant
dark rose


L amarque

Isa Biche

Iady Byron

Lutce or Smithi

Iandreth's Carmine

* La nymph

Miss Simpson

() $r$ lof

Nir Walter Scott

Foflatare large grecnish white-superb

flesh colour

brilliant pink, fine

light yellow

superb carmine, immense clusters

pale rose

suberb blush

brilliant pink

rosy lilac

rich ycllow .......... $\$ 1$

\section{ISLE DE BOURBON ROSES.}

'I'his family is likewise everblooming : most of them luave the fragrance of the garden rose, grow rapidly, and are perfectly hardy so far nortl as Philadelphia.

$$
50 \text { cents each. }
$$

Augustine Lelicur
Aristide
Emily Courticr
Gen. Duborg
Gloria de France
$\quad$ monthly cabbage
Gloria de Rosamond
IIcrmosa
Ienry Planticr
Ida Percot
Julia de Lyons
Jaeques
Ja Plicenix
Madam Iargat
$\quad$ Nerard
Marslal de Villiars
Neirum splendens
Ninion de l'linclos
Quecu
Theriseta
Thinocle

Augustine Itclicur

Gen. Duborg monthly cabbage

IIermosa

Ienry Planticr

Ida Percot

Julia de Lyons

Jacques

I,a Plownix

MIarslıal de Villiars

Neirum splendens

Ninion de l'linclos

Quecu

Theriseta

Thinnocle

dark rose
pale rose
rose, fine
rose
rose, very fragrant
very dark rose
light rose
rose red
lilac
white
dark red
bright red
dark rose
light rose
dark rose
rose, fragrant
rose
dark rose
deep blush
rose
rosy purple

dark rose

pale rose

rose, fine

rose

rose, very fragrant

very dark rose

light rose

rose red

lilac

white

dark red

bright red

dark rose

light rose

dark rosc

rose, fragrant

deep blush

rosy purple

\section{CLIMBING ROSES.}

Tliese fower annually in immense clusters, grow rapidly ind are quite hardy.

50 cents each-per dozen, $\$ 4$ to $\$ 6$.

Banksia lutca

alba double yellow

white 
Boursalt

\section{purpurea \\ blush \\ gracilis}

Bengalensis scandens

Fclicit de purpurea

Greville

Multaflora alba

Rubifolia clegans purpurea Queen alba

Russeliana

Sempervirens pleno

Triomph de Bollwylcr

Laura Da Voust rose colour

pirple-profuse

large blush

bright rose

large rosy white

blush white

Greville produces immensc clus-

$\{$ ters, of various eolours and

( shades, from white to crimson. pink

blush white

single miehigan or prairie

double pink

" purple

" pink

" blush white

crimson cottage rose

superb white

blush white

whitc

\section{MYCROPHYLLA ROSES.}

50 cents each.

Maria Leonida

Microphylla rosea

odorata alba white extra fine

rose eoleur

creamy white

\section{MUSK SCENTED ROSES.}

\section{0 cents each.}

Moschata

superba

Princess de Nassau white, semi-doublc

pure white, very double

whitc, double

\section{HARDY GARDEN ROSES.}

Miaulis

Coronation

Reme des Roses

Duc de Orleans

Painted damask

Brennus

Rivers' Geo. IV.

Iybrid blanch

Heureuse surprise

Ranunculus

La capricieuse

Royal Provins

Du Roi, perpetual rosy purple

purple crimson

bright crimson

dark rose

white

dark pink

superb crimson

white

earmine

purple, compaet

purple crimson

suberb pink

bright red
100

100

75

75

50

75

50

50

75

50

75

75

100 
IIarrisoni

Moss single

common

Luxemborg white

York and Laneaster

Prorins Belgic

Four Seasons

Moretti

Burgundy yellow Austrian briar crimson, very mossy

rose

crimson

perpetual

red and white

large pink

pink

light rose

rose, compact

\section{APPLES.}

25 cents each $\$ 20$ per hundred.

Those marked * are of Aincriean origin.

Those marked t are eelebrated for the table.

Astraclian red,

*Baldwin,

* Bevan,

* Bough, early,

*Carthouse or Gilpin,

Codlin English,

Crab Siberian, large,

*Cumberland spice,

*Doctor or Dewitt,

Double flowcring, ornamental

Early Queen,

* Early Harvest, Princes,

* Greening Burlington,

* tGreening Rhode Island,

IIaw thornden,

* Harrison, Harrison's Newark,

Juneating Red,

Lady A pple, Pomme d'Api.

Lady Finger,

* +Lippincott,

* †Maiden's Blush,

Morgan,

Pearmaine Blue Winter,

* Pearmaine Summer,

*Pennock's Red,

Pippin Bullock, Sheepnose,

*Pippin Golden,

* Pippin Miehael Henry,

* Pippin Ilolland or Fall,

Pippin Blcnhcim,

*Pippin, Green Newtown,

* +Pippin, Yellow Newtown,

Pippin Ribston,

* +Roxbury Russett,
Ripe from Aug. to Sept.

Nov. "Mar.

July " Aug.

do. do.

Dec. "Apl.

Aug. " Oct.

do. "Sept.

Oet. "Feb.

do. "Jan.

Suly " Aug.

do. do.

Oet. "Apl. do. "Jan.

Oet. "Mar.

July "Aug.

Nov. "Sept.

Oet. "Jan.

July " Aug.

Sept. "Jan.

Oet. "Jan.

Nov. "Jan.

Aug. "Sept.

Nov. "Mar.

Dec. "Mar.

Nov. "Fcb.

Nov, "Feb.

Oet. "Feb.

Nov. "Feb.

Nor. "June

Nov. "June

Oct. "Mar.

Dec. "Apl. 
Roman stem,

*Seek-no-further, Red,

* + Spitzenburg Kaighn's,

* Spitzenburg Newtowr,

* TSpitzcnburg Esopus,

* Sweeting Moore's,

* †Tewksbury Winter blush,

* Vandevere,

*Wine or Hay's Apple,

* Vinesap,

*Winter Quccn,

Yorkshire Grecning,

Ripe from Nov, to Feb.

Nov. " Feb.

Oct. " Dec.

Nov. "Jan.

Nov. "Mar.

Dec. "Apl.

Dec. "May

Dec. "Feb.

Nov." Peb.

Nov." Apl.

Nov, " Feb.

Oct. " Jan.

\section{PEARS.}

50 cents each.

'Thosc marked * are of Amcrican origin.

Bartlctt, William's Bonchretien,

Bcrgamot, Hampden's,

Bergamot, Autumn,

* Bleeker's Meadow,

Brrgamot, Easter,

Bergamot suisse longue panache,

Bucrre de Roi

Buerre D'iel

Buerre de Capaimont

Buerre d'Amalis

Buerre Bosc

Buerre summer

Bell Pear

* Bloodgood

*Chapman, Carr's

Doyenne Gris

Duchess d'Angouleme

Early Catharine

Green Chiscl Citron de Carmes

Gloux Marceau

* Heathcote

* Harvard

*Haddington Smith's

Holland Green Verte de Hollande

La Bon Cure

* Lodge

L'Echasseric-Walnut

*Lewis

Long Green Mouthwater-Mouille Bouche

Muscat Allemande

Passe Colmar

* Pennsylvania, Smith's

* Petre, Carr's

Ripe from Sept. to Oct.

Aug. "Sept.

Sept. " Oct.

Oct. "Dec.

Mal." Apl.

Oct. "Nov.

Sept. " Nov.

Oct. " Dec.

Sept. "Oct.

Sept. " Oct.

Sept. "Oct.

Aug. " Sep.

Oct. "Jan.

Aug. "Sept.

Sept. "Oct.

Sept. "Oct.

Oct. " Dec.

July " Aug.

Aug. " Sept

Nov. "Jan.

Sept. " Oct.

Sept. " Oct.

Sept. "Dec.

Oct. " Dec.

Sept. " Oct.

Oct. " Dec.

Nov, "Feb.

Oct. "Jan.

Sept. "Oct.

Nov. " Feb.

Nov, "Jan.

Sept. "Nor.

Sept. " Oct. 
* Rushmorc

Rousselet de Rheims-Musk or spice Pear

Sept. to Oct.

*Stevens' Gencssce

*Seckel

Sugar Pear

St. Germaine, Uvedales

*St. Germaine P'rince's

Swan's L'gg, Moorful Egg

Urbaniste

* Washington

Oet. "Nov

Aug. " Oct.

Sept." Oet.

Dec. " Apl.

Nov." Jan.

Oet. "Nov.

Oct. "Nov

Scpt." Oet.

\section{PLUMS.}

*Amcrican scedlings, of acknowledged merit-50 cents each.

Apricot plum

* Bingliam--yellow $\mathrm{egg}$

* Bolmar's Washington

*Blceker's Red

Blue Sugar

Blue Gagc

* Brevoort's Purple

*Blccker's Gage

Coe's Golden Drop

* Cooper's Red American

Damson yellow

Gage Green-Reine claude

Ripe in Augr.

Aug.

Sept.

Aug.

Aug.

Ang.

Sept.

Aur.

Sept.

Gage yellow

* Gage searlet

*Gage Sehuyler's

* Gagc Prinee's Imperial

Goliah, caledonia

"IIulings' superb

Magnum Bonum Red, Purple Egg

Magnam Bonum White-Ycllow Egg

Morocco, Black Damask

Nectarine, Prune Peche

Orleans, Red Orleans

Orleans Wilmot's New Early

Orleans Larly, Monsieur

Prune German, German Quetzc

Rivers' Seedling

* Verplanck's Purple

Niept.

A ug.

Aug.

Aug.

$\Lambda \mathrm{ug}$.

Aug.

Aug.

Ang.

Sept.

Sept.

Aug.

Aug.

Aug.

July

Aug.

sept.

sept.

Sept. 


\section{CHERRIES.}

Those marked, thus * arc suitable for preserves, tarts, \&c.

50 cents each, $\$ 40$ per hundred.

Amber Heart English

Belle de choisy

Biggarreau Napoleon

Biggareau White

Biggareau Red

Black Heart

Bleeding Heart

Blaek Eagle

Black Tartarian

* Carnation

Double flowering, ornamental

Downer's late Red

Downton

*Early Richmond, Morello

Herefordshire white Heart

Knight's Early Black

May-duke Early

Mayduke Latc

Mazzard Black

*Morello English

*Morello Frencl

*Morello Kentish

* Morello Plumstone

Ox Heart

White Heart

Yellow Spanish, Graffion

Ripc in June

June

July

July

July

June

June

June

June

Junc

July

July

June

June

June

June

July

July

July

July

July

July

June

Junc

July

\section{PEACHES.}

Those marked * are clingstones-25 cents each, $\$ 1875$ per hundrcd.

*Algiers Winter

Albcrgc, yellow rare ripe

Double Blossom, ornamantal

Grosse Mignone

Heath Frcestone

*IIcath Late

* Jemon Cling

Large Early, New York rave ripe

Large Early rare ripe

Malaeoton Red

Malaeoton White

IIorris' Red
Ripe in Sept.

Aug.

Aug.

Sept.

Oct.

Sept.

Aug.

July

Sept.

Aug.

Aug. 
QUINCES, \&c.

Morris' White

Nutmeg White

Nutmeg Red

* Oldmixon Cling

Oldmixon Freestone

President

Red Rarc Ripc

*Rodman's Cling

Smock's latc

Strawberry

*Tippecanoc

'Troth's Early Red

Waslington Frecstonc

Ward's Late

Aug.

July

July

Sept.

Sept.

Sept.

Aug.

Sept.

Sept.

Scpt.

Scpt.

Aug.

Aug.

Sept.

QUINCES-50 cents each.

Large Orange

Pear Shaped

CURRANTS-25 cents each.

Large dutch red

Large white crystal

English black

Missouri currant-ornamantal.

\section{RASPBERRIES.}

Red American or Genessce, (\$6 per 100)

Black American,

White Antwerp,

Red Antwerp,

New Red Antwerp,

Franconia,

(6 cents cach)

(10 cents each)

(10 cents cach)

(25 cents cach;

(20 cents each)

ALMONDS -37 i cents each.

Hard shcll,

Doublc flowering, ornamental.

Soft shell

GOOSEBERRIES-25 cents each.

20 Sclect European sorts. 
MULBERRIES-25 cents each.

Large blaek European

Red Ameriean

White Italian

\title{
FOREIGN GRAPES.
}

\section{0 cents each.}

Black Hamburg

Blaek Cluster

Blaek Frontignae

Black Lombardy

Blaek Frankindale

Clapier

Cliasselas "Neekerina"

Chasselas of Fontainbleau

Esperione

Grizly Frontignae

Gross Perle rose
Madeline

Miller's Burgundy

Museat of Lunel

Morillion Rouge

Noble Vernago

Purple Constantia

White Museat of Alexandria

White Gaseoigne

White Sweet Water

White Syrian

Yellow Provence, Orwigsburg.

\section{NATIVE GRAPES.}

\author{
50 cents each.
}

Bland's Virginia

Catawba

Elsinburg
Isabella

Longworth's Ohio "segar box"

Seuppernong

\section{STRAWBERRIES.}

Large Hudson, excellent, cultivated for the supply of the Philadelphia market

Hautbois, Alpine, delightful musky flavour

Hovey's Seedling, unsurpassed in quality and pro. ductiveness

Ross' Phønix, first quality, excellent flavour and very produetive

Keen's Seedling, very large and productive

Swainstone Seedling, frequently produces second crop in autumn

Per doz., per hund.

$\$$ cts. \$cts.

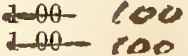

$37 \quad 200$

$50 \quad 300$

$25 \quad 150$

100 


\section{NUTS.}

Spanish Clicstnut English Filbert

American do. English Walnut large fruited each

50

100

Cob nut

"

Hazlenut large fruited "
37

25

\section{ASPARAGUS ROOTS.}

Pcr hundred $\$ 100$

Pcr thousand $\$ 750$

RHUBARB.

Victoria

'I'ubulsk

$\$ 100$ Mammoth

\section{S $\mathrm{EIICD}$}

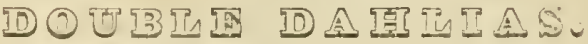

Alba Purpurea superba, Bates'-purple ground, tipped white.

Argo, Widnall's-ycllow eupped petals.

- Augustus, schmilz — dark purplc.

Admiral Stopford, Trentfield's-dark maroon.

Andrew IIofer, IIolmes'-dark marnon, cupped.

* Argus, Schmitz-primrose yellow tipped with rose.

* Alexander the Great, Schmitz-dark erimson finely cupped.

Bloomsbury, Lee's-large bright searlet, best scarlet out.

Bedfurd Surprise, sheppard's-rosy crimson.

Berkshire Chanpion, dark erimson.

Countess of Merton, Jeffrey's-scarlet, fine form.

Cleopatra-white, fine flower.

Cambridge Jero, Wiluall's-dark maroon.

${ }^{*}$ Calcb Cope, Schmitz-mottled rose, finc.

Defiance, Hor eood's-fine purple.

Diana, Elphinstone's-yellow and crimson.

Duchess of Richmond, Llphinstone's-salmon.

Duke of Bedford, nearly black.

Donna Antoni, Derlin's-rosy purple.

Elizabeth, Tientficid's-white, mottled with purple.

Exquisite, Givliug s - fine salmon.

England's Woider, Halton-white spotted with lilac.

Esscx 'Trimmph, T'm ville-dark maroon.

Fire Ball, Squilss-virid scarlet

"Fanny, Ileiskill's-pure white, tipped lilac.

* Fire King, Schmilz-bright scarlet.

Glory of Ply'moutli, Rendle's-white, purple tipped.

"IIcro of 'T'ippecanoe, Ilancock's-rosy purple. 
Hope, Neville-superb rose.

*Horace Binney, Schmitz-shaded maroon.

*Henry Clay, Schmitz-dark claret, fine form.

* Josephine, Schmitz-pure white; laced with purple.

*Louisa, Schmitz-salmon.

* Lady Washington, Schmitz-pure white, lilac tinge.

Lady Ashburton, Russell-blush white.

Lady Webster, Knight-crimson.

La Carnation, Paul's-white, flaked with rose.

Le Grand Baudin, Low-shaded crimson.

* Miss Percival, Schmitz-fine white.

*Miss McCausland, Schmitz-fine dark rose.

*Miss Carpentier, Schmitz-mottled rose, fine.

*Mrs. Rushton, Buist-pure white tipped with rose.

*Mary Ann, Schmitz-pure whitc, fine.

Maid of Bath, Davis'-pure white, edge purple.

Marshal Soult, Elphinstone's-lilac and red.

*Mrs. IIibbert, Schmitz-blush rose, fine form.

Miranda, Brown's-blush white tipped with rose.

Northern Beauty, Robinson's-white, tipped with rose.

Ne Plus Ultra, Widnall's-purple and crimson.

Nigra et Alba, Girling-white, edged with purple.

Oakley's Surprise-purple, tipped with white.

*Pocahontas, Schmilz-vermillion, fine shape.

Prince of Wales, Dodd's-splendid ycllow.

Princess Royal, Hudson's-pale amber, fine.

Pandora, Bowman-crimson, fine form.

Quecn of Beauty-lilac, fine form.

Queen, Widnall's-peach blossom.

Quilled Perfection-rich crimson.

Reine de Fecs, Girling-crimson and white.

Royal Standard, Whales-rosy purple.

Rienzi, Widnall's—crimson and maroon.

Reliance, Widnall's-bright orange.

Striata Formosissima, Bates'-white, crimson and maroon.

Sir F. Johnstone, Hillier-rosy crimson, superb.

Springfield Major-rosy crimson.

Sir Henry Fletcher-rosy crimson.

*Schmitz's General Washington--rich, sliaded red.

* " Negro-shaded maroon.

* " Rival-maroon.

* “ Conqueror-very dark maroon.

Thomas Clarkson, Smith-rosy purple.

*Thomas C. Percival, Schmitz-white, very fine.

Unique, Ansell's-yellow, tipped with bronze.

*Yellow Victory, Schmitz - brimstone yellow, finc.

Assortments-12 very hest sorts, $\$ 6 ; 12$ very finc, $\$ 4.50 ; 12$ finc, $\$ 2.50$, and at the same rate for single roots.

* American Seedling varieties of established reputation. 


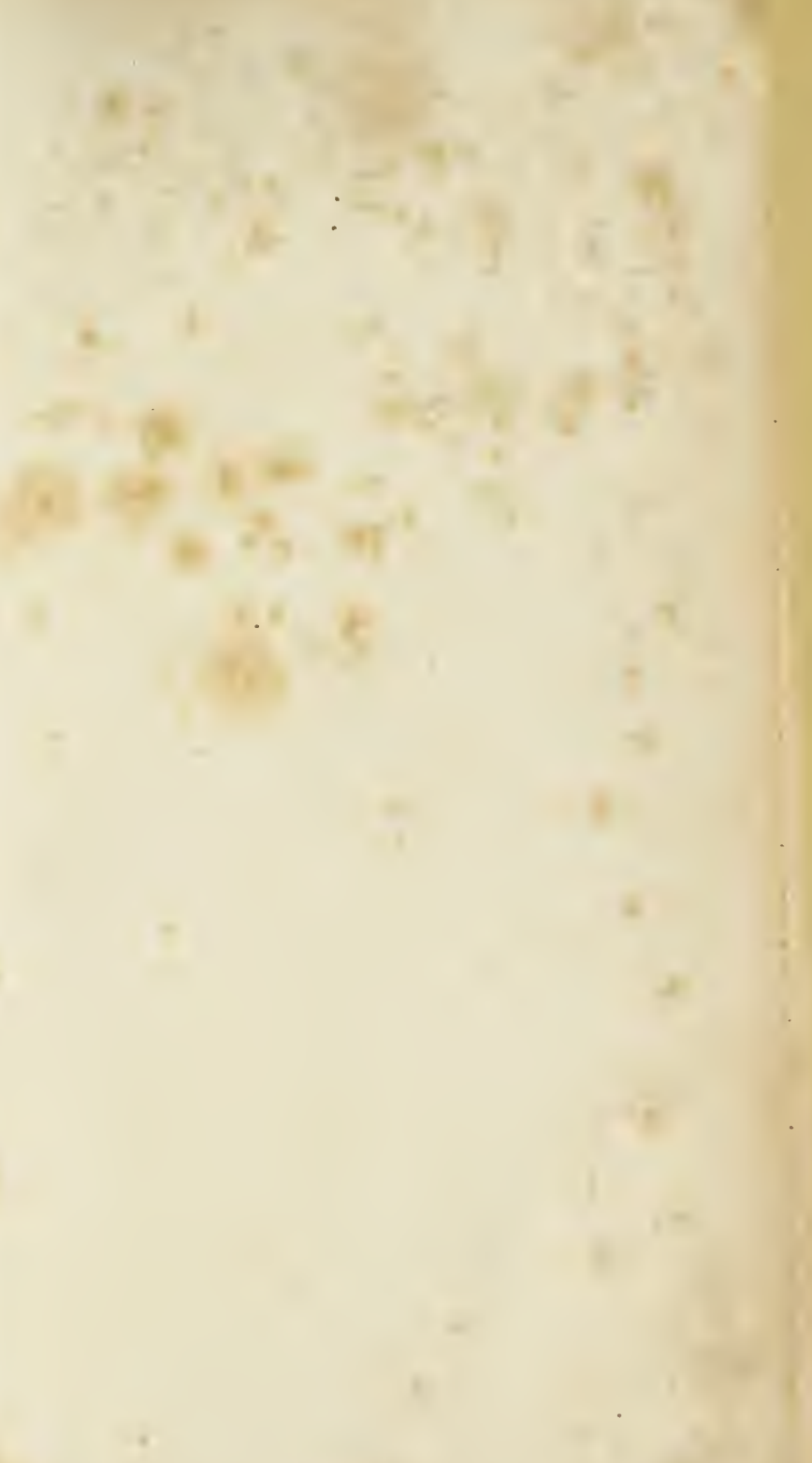




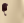





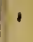

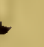

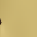


